\title{
Identification of $n n p$ and npp Auger recombination as significant contributor to the efficiency droop in (Galn)N quantum wells by visualization of hot carriers in photoluminescence
}

\author{
M. Binder, ${ }^{1, a)}$ A. Nirschl, ${ }^{1,2}$ R. Zeisel, ${ }^{1}$ T. Hager, ${ }^{1}$ H.-J. Lugauer, ${ }^{1}$ M. Sabathil, ${ }^{1}$ \\ D. Bougeard, ${ }^{2}$ J. Wagner, ${ }^{3}$ and B. Galler ${ }^{1}$ \\ ${ }^{1}$ OSRAM Opto Semiconductors GmbH, Leibnizstraße 4, 93055 Regensburg, Germany \\ ${ }^{2}$ Institut für Experimentelle und Angewandte Physik, Universität Regensburg, Universitätsstraße 31, \\ 93040 Regensburg, Germany \\ ${ }^{3}$ Fraunhofer-Institut für Angewandte Festkörperphysik, Tullastraße 72, 79108 Freiburg, Germany
}

(Received 17 May 2013; accepted 31 July 2013; published online 15 August 2013)

\begin{abstract}
We report the direct observation of hot carriers generated by Auger recombination via photoluminescence spectroscopy on tailored (AlGaIn)N multiple quantum well $(\mathrm{QW})$ structures containing alternating green and ultra-violet (UV) emitting (GaIn)N QWs. Optically pumping solely the green QWs using a blue emitting high power laser diode, carrier densities similar to electrical light-emitting diode (LED) operation were achieved, circumventing possible leakage and injection effects. This way, luminescence from the UV QWs could be observed for excitation where the emission from the green QWs showed significant droop, giving direct evidence for Auger generated hot electrons and holes being injected into the UV QWs. An examination of the quantitative relation between the intensity of the UV luminescence and the amount of charge carriers lost due to drooping of the QWs supports the conclusion that Auger processes contribute significantly to the droop phenomenon in (AlGaIn)N based light-emitting diodes. (c) 2013 AIP Publishing LLC. [http://dx.doi.org/10.1063/1.4818761]
\end{abstract}

Due to their high lifetimes and efficiencies along with rapidly declining prices, light-emitting diodes (LEDs) based on (AlGaIn)N multiple quantum well (MQW) structures are on their way to replace incandescent as well as fluorescent lighting. Despite great progress in recent years, resulting in peak power conversion efficiencies of up to $81 \%,{ }^{1}$ one obstacle still to overcome is the decrease in efficiency towards high operating current densities, a phenomenon commonly known as droop. ${ }^{2,3}$ The current dependency of the internal quantum efficiency (IQE) can be modeled in good quantitative agreement with experimental data using an $A B C$ rate equation model ${ }^{4-6}$

$$
\mathrm{IQE}=\frac{B n^{2}}{A n+B n^{2}+C n^{3}},
$$

where $n$ is the electron density which is equal to the hole density $p . A$ and $B$ are the coefficients of Shockley-ReadHall (SRH) and radiative recombination, respectively. ${ }^{7,8}$ In this model, the drop in efficiency towards high current densities is ascribed to a loss process proportional to $n^{3}$, represented by the recombination coefficient $C$. Due to the cubic dependency on the charge carrier density under electroluminescence as well as under photoluminescence (PL) conditions, Auger recombination in the active layer is one of the main suspects to be the cause of the drop in efficiency. ${ }^{4,5,9}$ Yet, using microscopic calculations, Hader et al. simulated the magnitude of direct Auger recombination in the (AlGaIn)N material system and concluded that Auger losses are too small to account for the droop. ${ }^{10}$ Taking phononassisted Auger recombination into account, Kioupakis et al.

a)Electronic mail: michael.binder@osram-os.com were able to motivate higher $C$ values which are in good agreement with experiment. ${ }^{6,11}$ Nevertheless, the origin of the droop is still debated controversially. Other possible explanations, like recombination at dislocations ${ }^{12}$ or electron leakage enhanced by high internal piezoelectric fields, ${ }^{13-16}$ have also been suggested as the root cause for the drop in high current efficiency. Only recently, Iveland et al. were able to provide evidence for Auger recombination in an electrically driven (GaIn)N based LED by detecting hot electrons. ${ }^{17}$ In their experiment, they claim to visualize electronelectron-hole processes with a ratio of $10^{-6}$ between detected Auger current and current lost due to droop. Yet, the conclusions drawn from these experiments are currently subject to controversial discussion. ${ }^{18}$

In this letter, we prove that both electron-electron-hole ( $n n p)$ as well as electron-hole-hole (npp) Auger processes contribute to the droop phenomenon in (GaIn)N QWs using a PL based approach visualizing approximately $1 \%$ of the droop current. Exciting a test structure composed of alternating ultra-violet (UV) and green emitting QWs with a blue laser diode, we exploit the fact that in an Auger process energy, gained from band-to-band recombination, is transferred to a third charge carrier. As the resulting hot charge carriers have an energy of approximately one band gap above the respective band edge, there is a finite probability that they relax into UV QWs (see Fig. 1). Driving the green quantum wells into the droop regime, it is possible to detect ultra-violet luminescence if both Auger electrons and holes are generated as proposed by Kioupakis et al. ${ }^{11}$ We observe such UV luminescence under excitation with photons of an energy high enough to generate electron-hole pairs in the green QWs, but low enough not to excite the UV wells directly. 
The undoped PL samples were grown in $c$-direction on sapphire substrates by metal-organic vapour phase epitaxy. The deposition started with a low-temperature GaN nucleation layer followed by a $4 \mu \mathrm{m}$ thick GaN buffer. Next, the active region, differing in design for 3 different samples, was grown (see Fig. 1). Sample G + UV consists of five green and five UV QWs in alternating order. The two reference samples composed solely of green (sample G) and UV (sample UV) quantum wells, respectively. 006-reflex XRD measurements were carried out on all samples, yielding distinct peaks suggesting good material quality. By comparing these measurements with XRD-simulations, the indium content of the green and $\mathrm{UV}\left(\mathrm{In}_{x} \mathrm{Ga}_{1-x}\right) \mathrm{N}$ based QW-layers was determined to be $22 \%$ and $10 \%$, respectively. To suppress the escape of carriers from the (GaIn)N QWs by tunnel processes or carrier spill-over, all QW layers were spatially separated by $7 \mathrm{~nm}$ thick $\left(\mathrm{Al}_{0.2} \mathrm{Ga}_{0.8}\right) \mathrm{N}$ barriers. Epitaxial growth was concluded with a $5 \mathrm{~nm} \mathrm{GaN}$ cap.

Optical characterization was performed in a helium closed cycle cryostat. To ensure low thermal resistance, all samples were glued on a copper cooling finger using conductive silver paste. Photoluminescence measurements were carried out on all samples at $12 \mathrm{~K}$ using two different excitation sources. First, the emission spectra of all samples were measured using a $\mathrm{HeCd}$-laser emitting at $325 \mathrm{~nm}$ with an excitation density of $\approx 50 \mathrm{~W} / \mathrm{mm}^{2}$ (see Fig. 2). As expected from the epitaxial designs, two (GaIn)N-related peaks are present in the emission spectrum of sample G + UV, while only single peaks are observed in sample $G$ and sample UV. Whereas the peak intensity of the emission originating from the UV wells is at $390 \mathrm{~nm}$, the green quantum wells exhibit a broader emission centered at $540 \mathrm{~nm}$. Considering the similar luminescence of the respective QWs, samples G and UV are suitable references.

In order to demonstrate the existence of hot electrons and holes originating from Auger recombination via ultraviolet luminescence, we excited only the green QWs using a high power blue diode laser. With an output power of $P_{450 \mathrm{~nm}}=1.2 \mathrm{~W}$ and emission wavelength of $450 \mathrm{~nm}$ at a driving current of $1 \mathrm{~A}$, charge carrier densities similar to those generated in electrically driven LEDs are achieved. ${ }^{19}$ To prevent resonant excitation of UV QWs, a long pass filter was mounted in front of the laser diode, blocking the short-

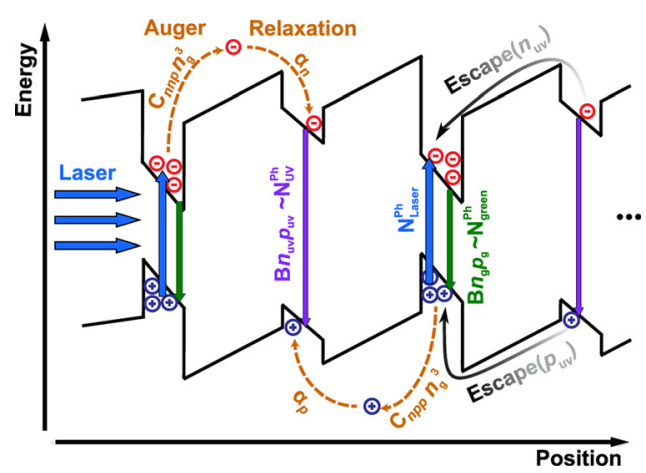

FIG. 1. Schematic drawing of the band structure of sample G + UV. Ultraviolet quantum wells are used to capture hot charge carriers generated by Auger processes in the green wells. Luminescence originating from the UV wells therefore visualizes Auger recombination in the green wells.

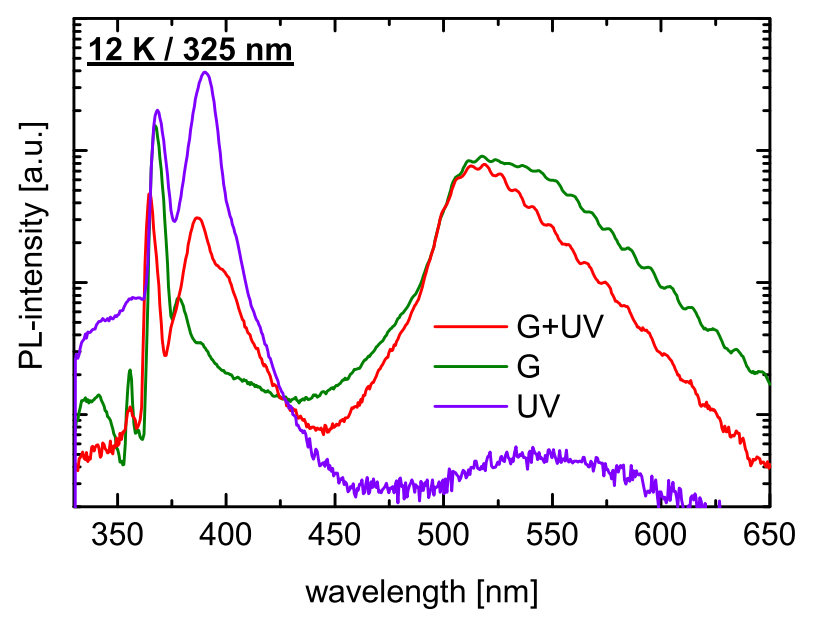

FIG. 2. Photoluminescence spectra of samples G + UV, G, and UV at $12 \mathrm{~K}$ under excitation with a wavelength of $325 \mathrm{~nm}$.

wavelength spontaneous emission of the excitation source. Furthermore, the whole setup was optimized to minimize stray light. A notch filter in front of the spectrometer was utilized to block the main emission of the excitation laser. The resulting residual spectrum, depicted as "laser", is shown in Fig. 3 along with measured PL spectra of the different samples. While the luminescence in the regions marked with $\mathrm{N}_{\mathrm{UV}}^{\mathrm{Ph}}$ and $\mathrm{N}_{\text {green }}^{\mathrm{Ph}}$ can be ascribed to the respective QWs, the spectral range from $420 \mathrm{~nm}$ to $500 \mathrm{~nm}$ is dominated by remnant spontaneous and stimulated emission of the laser diode. In accordance to the mechanism described in Fig. 1 for sample $\mathrm{G}+\mathrm{UV}$, besides luminescence of the green QWs, a strong UV signal similar to the emission spectrum upon $325 \mathrm{~nm}$ excitation of the UV QWs can be observed, indicating the generation of hot charge carriers due to Auger recombination.

However, one might argue that UV emission can be caused by other effects, such as V-Pits in the green QWs, thermal or surface potential effects as well as direct twophoton absorption. Another possible mechanism generating hot charge carriers, hence another possible origin for the UV luminescence, may be free carrier absorption ${ }^{20}$ of the

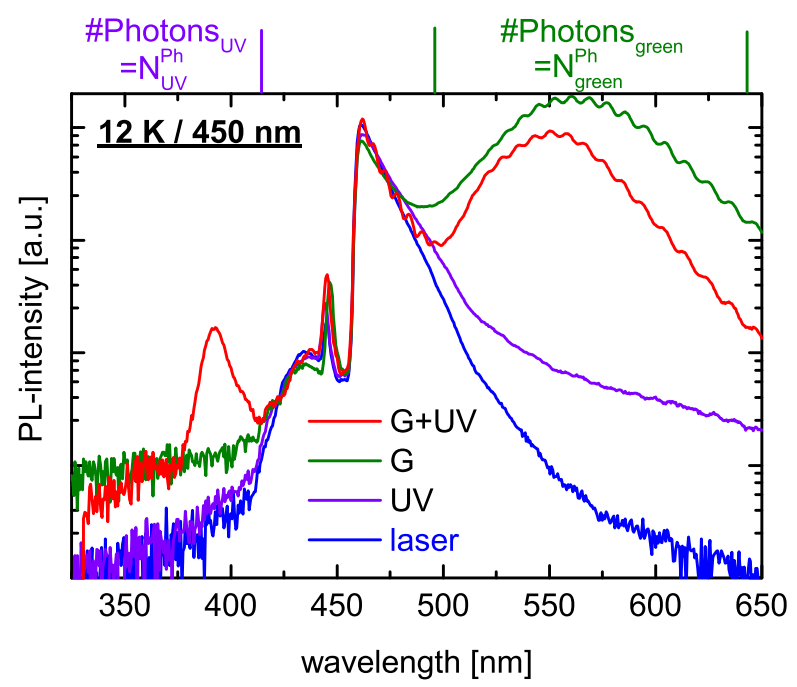

FIG. 3. Photoluminescence spectra of samples G $+\mathrm{UV}, \mathrm{G}$, and UV at $12 \mathrm{~K}$ under excitation with a wavelength of $450 \mathrm{~nm}$. 
pumping laser photons by the charge carriers in the green wells. Further investigations were done to clarify the origin of this UV emission.

Since the reference sample G, composed solely of green QWs, does not show any ultra-violet photoluminescence, the UV peak of sample $\mathrm{G}+\mathrm{UV}$ must result from radiative charge carrier recombination in the UV wells and is not an artifact of the green QWs itself such as V-Pit emission.

Direct excitation of the UV wells can be excluded by analyzing sample UV. The PL measurement of this test structure yields almost the same spectrum as the reference measurement of the direct laser emission (Fig. 3). Only for wavelengths exceeding $460 \mathrm{~nm}$, minor additional PL intensity is observed, which can be ascribed to the well-known yellow luminescence. ${ }^{21}$ The absence of further PL intensity in the UV region ensures that the UV peak in sample G + UV is not caused by direct excitation of the UV QWs via two-photon absorption.

Temperature-dependent measurements were performed to investigate whether the generation of charge carrier density in the UV QWs is related to thermal filling caused by unintentional heating of the sample at high excitation power densities. Varying the temperature from $12 \mathrm{~K}$ to $50 \mathrm{~K}$ had no impact on the intensity of the UV peak. Due to the strong temperature dependency of SRH-recombination, ${ }^{22,23}$ a further increase in temperature led to a rapid decay in UV intensity. Therefore, thermal effects can be excluded as the cause of the UV luminescence.

Furthermore, samples similar to G + UV and UV were grown with a $100 \mathrm{~nm}$ GaN cap. Since they yield similar PL spectra, surface potential effects can also be eliminated as a possible cause for charge carrier generation in the UV wells.

Utilizing the fact that the charge carrier density of the green QWs does not scale linearly with the PL excitation power, one can distinguish between charge carrier excitation linked to Auger effect or free carrier absorption. We varied the PL excitation power over four orders of magnitude to determine the correlation between the number of incident laser photons $\left(\mathrm{N}_{\text {laser }}^{\mathrm{Ph}}\right)$ and the intensity of the green $\left(\mathrm{N}_{\text {green }}^{\mathrm{Ph}}\right)$ as well as $\mathrm{UV}\left(\mathrm{N}_{\mathrm{UV}}^{\mathrm{Ph}}\right)$ emission (for assignment see Fig. 3). Since electrons and holes in all green QWs are generated in pairs by absorption of laser photons, we can assume $n_{\mathrm{g}} \cong p_{\mathrm{g}}$, where $n_{\mathrm{g}}$ is the electron and $p_{\mathrm{g}}$ is the hole density in the green wells, respectively. The generation rates $G_{n, \text { uv }}$ and $G_{p, \text { uv }}$ of electrons $\left(n_{\text {uv }}\right)$ and holes $\left(p_{\text {uv }}\right)$ in the UV wells by Auger processes in the green QWs can be expressed as function of $n_{\mathrm{g}}$

$$
G_{n, \mathrm{uv}}=\alpha_{n} \times C_{n n p} \times n_{\mathrm{g}} \times n_{\mathrm{g}} \times p_{\mathrm{g}} \cong \alpha_{n} \times C_{n n p} \times n_{\mathrm{g}}{ }^{3}
$$

and

$$
G_{p, \text { uv }}=\alpha_{p} \times C_{n p p} \times n_{\mathrm{g}} \times p_{\mathrm{g}} \times p_{\mathrm{g}} \cong \alpha_{p} \times C_{n p p} \times n_{\mathrm{g}}{ }^{3},
$$

where $C_{n n p}$ and $C_{n p p}$ are the recombination coefficients for nnp and npp Auger processes, respectively. $\alpha_{n} / \alpha_{p}$ are the capture probabilities for hot electrons/holes by the UV wells. Due to the fact that UV luminescence remains unaffected by a temperature increase from $12 \mathrm{~K}$ to $50 \mathrm{~K}$, we conclude that SRH recombination in the UV QWs is negligible at $12 \mathrm{~K}$. Considering the low intensity of the UV emission throughout the whole measurement range compared to the green luminescence, droop effects in the UV well can also be neglected, making radiative recombination the only recombination path in the UV wells. Hence, in steady state condition, where charge carrier generation is equal to recombination and escape, the rate equations can be simplified to

$$
B_{\mathrm{uv}} \times n_{\mathrm{uv}} \times p_{\mathrm{uv}}=\alpha_{n} \times C_{n n p} \times n_{\mathrm{g}}{ }^{3}-\operatorname{Escape}\left(n_{\mathrm{uv}}\right)
$$

and

$$
B_{\mathrm{uv}} \times n_{\mathrm{uv}} \times p_{\mathrm{uv}}=\alpha_{p} \times C_{n p p} \times n_{\mathrm{g}}{ }^{3}-\operatorname{Escape}\left(p_{\mathrm{uv}}\right),
$$

where $\operatorname{Escape}\left(n_{\text {uv }}\right)$ and $\operatorname{Escape}\left(p_{\text {uv }}\right)$ represent an escape mechanism for electrons and holes from the UV wells, respectively (see Fig. 1). The charge carrier densities in the UV QWs are governed by

$$
\left(\alpha_{n} C_{n n p}-\alpha_{p} C_{n p p}\right) n_{\mathrm{g}}{ }^{3}=\operatorname{Escape}\left(n_{\mathrm{uv}}\right)-\operatorname{Escape}\left(p_{\mathrm{uv}}\right) .
$$

As Eq. (6) shows, $\left[\operatorname{Escape}\left(n_{\text {uv }}\right)-\operatorname{Escape}\left(p_{\text {uv }}\right)\right]$ must scale with $n_{\mathrm{g}}{ }^{3}$. Since, for this to be valid under all conditions, any other dependency on $n_{\mathrm{g}}$ but $n_{\mathrm{g}}{ }^{3}$ of the individual loss mechanisms must be identical in both escape terms, we conclude that $\operatorname{Escape}\left(n_{\mathrm{uv}}\right)$ as well as $\operatorname{Escape}\left(p_{\mathrm{uv}}\right)$ also scale with $n_{\mathrm{g}}{ }^{3}$. Hence, it can be seen from Eqs. (4) and (5), UV luminescence provoked by Auger processes in green QWs is proportional to the cube of the carrier density in the green QWs

$$
\mathrm{N}_{\mathrm{UV}}^{\mathrm{Ph}} \propto B_{\mathrm{uv}} \times n_{\mathrm{uv}} \times p_{\mathrm{uv}} \propto n_{\mathrm{g}}{ }^{3} .
$$

By contrast, UV light stimulated by free carrier absorption in the green well is expected to scale with $\mathrm{N}_{\text {laser }}^{\text {Ph }} \times n_{\mathrm{g}}$. To check which dependency of $\mathrm{N}_{\mathrm{UV}}^{\mathrm{Ph}}$ on $n_{\mathrm{g}}$ prevails, we normalized $\mathrm{N}_{\mathrm{UV}}^{\mathrm{Ph}}$ to the respective model, namely, free carrier absorption $\left(\propto \mathrm{N}_{\text {laser }}^{\mathrm{Ph}} \times n_{\mathrm{g}}\right)$ and Auger $\left(\propto n_{\mathrm{g}}^{3}\right)$, which is shown in Fig. 4. Since the radiative recombination rate of the green well is proportional to its electron and hole density $\left(n_{\mathrm{g}} \propto \sqrt{\mathrm{N}_{\text {green }}^{\mathrm{Ph}}}\right)$, the dependency of $n_{\mathrm{g}}$ on excitation power was extracted from measured green intensity. As the ratio is constant for the Auger model, while it decreases with $\mathrm{N}_{\text {laser }}^{\mathrm{Ph}}$ for the free

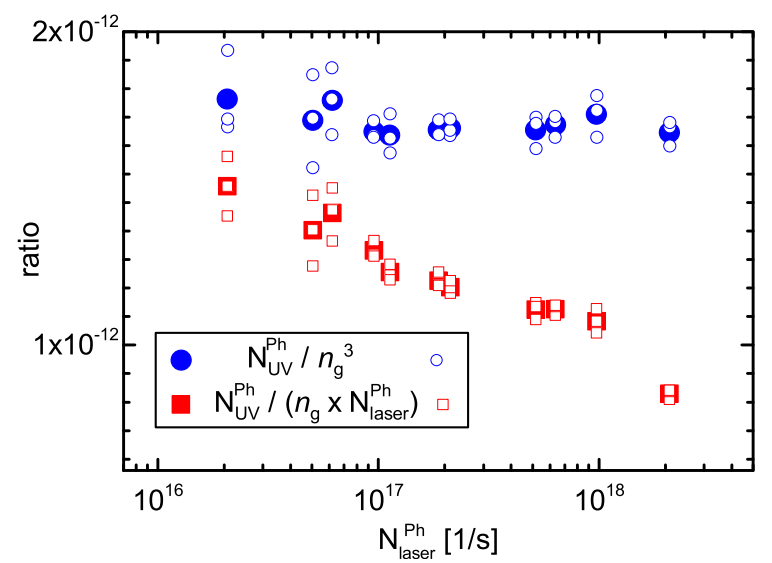

FIG. 4. Dependency of $\mathrm{N}_{\mathrm{UV}}^{\mathrm{Ph}} / n_{\mathrm{g}}^{3}$ and $\mathrm{N}_{\mathrm{UV}}^{\mathrm{Ph}} /\left(\mathrm{N}_{\text {laser }}^{\mathrm{Ph}} \times n_{\mathrm{g}}\right)$ on pump photon rate $\left(\mathrm{N}_{\text {laser }}^{\mathrm{Ph}}\right)$. Filled symbols are averaged over three single measurements represented by hollow symbols. 
carrier absorption model, we conclude that the UV luminescence is caused by Auger processes and not by free carrier absorption.

The discussion so far has revealed that both $n n p$ as well as $n p p$ Auger processes occur at a relevant rate in (GaIn)NQWs under the presence of carrier densities reached under LED operating conditions. To finally demonstrate the significance of those processes for the droop phenomenon, a quantitative analysis of the UV intensity with respect to the amount of current lost due to nonradiative recombination is performed. For this purpose, the ratio between excitation power and green photon flux as a measure for the IQE of the green QWs is displayed in Fig. 5. Due to the freeze-out of non-radiative recombination centers, the IQE remains constant for the low excitation regime.

Towards higher excitation intensities, a drop in efficiency can be observed. As Auger recombination, visualized by UV emission $\mathrm{N}_{\mathrm{UV}}^{\mathrm{Ph}}$, as well as the decrease in efficiency scale with $n_{\mathrm{g}}^{3}$, the relation $C \times n_{\mathrm{g}}^{3}=\beta \times \mathrm{N}_{\mathrm{UV}}^{\mathrm{Ph}}$ holds, where $\beta$ is a constant (see Fig. 5). In order to demonstrate that Auger recombination is the main contributor to droop, a quantitative discussion of $\beta$ is essential. Assuming that Auger processes are fully responsible for the drop in efficiency, $\beta$ can be expressed as

$$
\beta=\frac{C_{n n p}+C_{n p p}}{\min \left[\alpha_{n} \times C_{n n p}, \alpha_{p} \times C_{n p p}\right]},
$$

where $C_{n n p}+C_{n p p}$ reflects the overall Auger recombination rate and $\alpha_{n} \times C_{n n p}$ as well as $\alpha_{p} \times C_{n p p}$ are the generation rates for electrons and holes in the UV wells, respectively. As Eq. (8) shows, the intensity of the UV luminescence is controlled by the smaller of the two rates. Since Auger recombination generates one hot charge carrier per annihilated electron-hole-pair, for the case of symmetrical Auger $\left(C_{n n p}=C_{n p p}\right)$ and assuming that every hot charge carrier relaxes into the UV QWs $\left(\alpha_{n}=\alpha_{p}=1\right), \beta$ would reach the smallest possible value of 2 . Experimentally, we obtain $\beta \cong$ 200 and can thus establish that at least $1 \%$ of the droop can be ascribed to Auger processes.

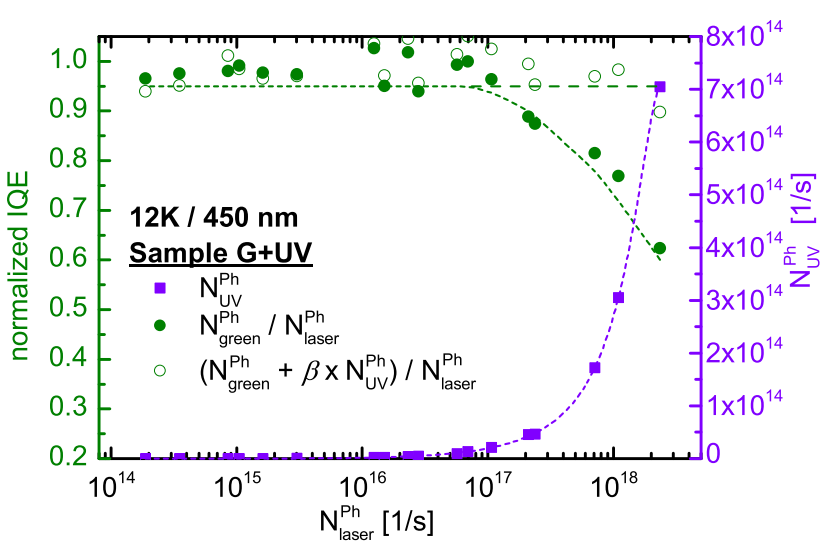

FIG. 5. As measure for the IQE of the green QWs, the ratio between green luminescence $\mathrm{N}_{\text {green }}^{\mathrm{Ph}}$ and excitation power $\mathrm{N}_{\text {laser }}^{\mathrm{Ph}}$ is plotted versus $\mathrm{N}_{\text {laser }}^{\mathrm{Ph}}$. Simultaneously with the onset of droop, a steep rise in intensity of UV emission can be observed. Replacing $\mathrm{N}_{\text {green }}^{\mathrm{Ph}}$ by $\mathrm{N}_{\text {green }}^{\mathrm{Ph}}+\beta \times \mathrm{N}_{\mathrm{UV}}^{\mathrm{Ph}}$ droop can be compensated.
However, it is obvious that a realistic percentage is much higher because of the following three reasons:

(1) As the green QWs constitute the energetic minimum of the structure, these layers are more probable to capture charge carriers.

(2) Hot electrons and holes are generated in the green QWs, which are spatially separated from the UV QWs. Therefore, these carriers have to overcome a significant distance in real space to be captured by the UV wells. As a consequence of (1) and (2), the relation $\alpha_{n}, \alpha_{p} \ll 1$ for the capture probabilities holds.

(3) Any asymmetry in the coefficients $C_{n n p} \neq C_{n p p}$, for which various ratios are theoretically obtained, ${ }^{1,24}$ leads to a further increase of the expected $\beta$ beyond the theoretical lower limit 2.

Due to these reasons, obtaining $\beta \cong 200$ from experiment shows that at least a considerable fraction of droop is caused by Auger processes.

In summary, a test structure composed of alternating ultra-violet and green quantum wells was pumped into droop regime using a blue high power laser diode. Taking advantage of the fact that energy gained in Auger band-to-band recombination is transferred to a third charge carrier, we were able to visualize Auger processes by emission of ultraviolet luminescence. Since for the ultra-violet luminescence hot electrons as well as hot holes are required, our experiment proves that both types of Auger processes ( $n n p$ as well as $n p p$ ) are relevant for the drop in efficiency. Using this resonant PL experiment, we can exclude loss channels caused by current injection and leakage. Previously, it has been shown that droop is a QW internal loss process exhibiting the same dependency on charge carrier density as Auger recombination ${ }^{4,5,9,17,19}$ and that first-principles calculations of Auger coefficients yield results that can quantitatively explain the drop in efficiency. ${ }^{11}$ Our detailed discussion of the relationship between UV intensity and amount of lost charge carriers proves that at least $1 \%$ of droop can be ascribed to Auger processes, with the realistic percentage likely to be higher. These findings further support the thesis that Auger recombination constitutes a significant role for the droop phenomenon in (AlGaIn)N based light-emitting diodes.

${ }^{1}$ Y. Narukawa, M. Ichikawa, D. Sanga, M. Sano, and T. Mukai, J. Phys. D: Appl. Phys. 43, 354002 (2010).

${ }^{2}$ T. Mukai, M. Yamada, and S. Nakamura, Jpn. J. Appl. Phys., Part 1 38, 3976 (1999).

${ }^{3}$ J. Piprek, Phys. Status Solidi A 207, 2217 (2010).

${ }^{4}$ Y. C. Shen, G. O. Mueller, S. Watanabe, N. F. Gardner, A. Munkholm, and M. R. Krames, Appl. Phys. Lett. 91, 141101 (2007).

${ }^{5}$ A. David and N. F. Gardner, Appl. Phys. Lett. 97, 193508 (2010).

${ }^{6}$ B. Galler, P. Drechsel, R. Monnard, P. Rode, P. Stauss, S. Froehlich, W. Bergbauer, M. Binder, M. Sabathil, B. Hahn, and J. Wagner, Appl. Phys. Lett. 101, 131111 (2012).

${ }^{7}$ W. Shockley and W. T. Read, Jr., Phys. Rev. 87, 835 (1952).

${ }^{8}$ R. N. Hall, Phys. Rev. 87, 387 (1952).

${ }^{9}$ A. Laubsch, M. Sabathil, J. Baur, M. Peter, and B. Hahn, IEEE Trans. Electron Devices 57, 79 (2010).

${ }^{10}$ J. Hader, J. V. Moloney, B. Pasenow, S. W. Koch, M. Sabathil, N. Linder, and S. Lutgen, Appl. Phys. Lett. 92, 261103 (2008).

${ }^{11}$ E. Kioupakis, P. Rinke, K. T. Delaney, and C. G. Van de Walle, Appl. Phys. Lett. 98, 161107 (2011). 
${ }^{12}$ A. Hangleiter, F. Hitzel, C. Netzel, D. Fuhrmann, U. Rossow, G. Ade, and P. Hinze, Phys. Rev. Lett. 95, 127402 (2005).

${ }^{13}$ J. Xu, M. F. Schubert, A. N. Noemaun, D. Zhu, J. K. Kim, E. F. Schubert, M. H. Kim, H. J. Chung, S. Yoon, and C. Sone, Appl. Phys. Lett. 94, 011113 (2009).

${ }^{14}$ M. H. Kim, M. F. Schubert, Q. Dai, J. K. Kim, E. F. Schubert, J. Piprek, and Y. Park, Appl. Phys. Lett. 91, 183507 (2007).

${ }^{15}$ M. F. Schubert, J. Xu, J. K. Kim, E. F. Schubert, M. H. Kim, S. Yoon, S. M. Lee, C. Sone, T. Sakong, and Y. Park, Appl. Phys. Lett. 93, 041102 (2008).

${ }^{16}$ M. F. Schubert, S. Chhajed, J. K. Kim, E. F. Schubert, D. D. Koleske, M. H. Crawford, S. R. Lee, A. J. Fischer, G. Thaler, and M. A. Banas, Appl. Phys. Lett. 91, 231114 (2007).

${ }^{17}$ J. Iveland, L. Martinelli, J. Peretti, J. S. Speck, and C. Weisbuch, Phys. Rev. Lett. 110(17), 177406 (2013).
${ }^{18}$ F. Bertazzi, M. Goano, X. Zhou, M. Calciati, G. Ghione, M. Matsubara, and E. Bellotti, e-print arXiv:cond-mat/1305.2512.

${ }^{19}$ B. Galler, M. Sabathil, A. Laubsch, T. Meyer, L. Hoeppel, G. Kraeuter, H. Lugauer, M. Strassburg, M. Peter, A. Biebersdorf, U. Steegmueller, and B. Hahn, Phys. Status Solidi C 8, 2369 (2011).

${ }^{20}$ O. Ambacher, W. Rieger, P. Ansmann, H. Angerer, T. D. Moustakas, and M. Stutzmann, Solid State Commun. 97, 365 (1996).

${ }^{21}$ D. M. Hofmann, D. Kovalev, G. Steude, B. K. Meyer, A. Hoffmann, L. Eckey, R. Heitz, T. Detchprom, H. Amano, and I. Akasaki, Phys. Rev. B 52(23), 16702 (1995).

${ }^{22}$ A. Hangleiter, D. Fuhrmann, M. Grewe, F. Hitzel, G. Klewer, S. Lahmann, C. Netzel, N. Riedel, and U. Rossow, Phys. Status Solidi A 201, 2808 (2004).

${ }^{23}$ J. Abell and T. D. Moustakas, Appl. Phys. Lett. 92, 091901 (2008).

${ }^{24}$ R. Vaxenburg, E. Lifshitz, and A. L. Efros, Appl. Phys. Lett. 102, 031120 (2013). 